\title{
Bilateral frontal intracranial xanthoma associated with type II hyperlipidemia in a 42-year-old woman: illustrative case
}

\author{
*Donny Argie, MD, Christopher Lauren, MD, and Elric B. Malelak, MD \\ Department of Neurosurgery, Prof. Dr. W. Z. Johannes Kupang Regional General Hospital, Kupang, East Nusa Tenggara, Indonesia
}

BACKGROUND Xanthoma is a granulomatous lesion that develops from leakage of circulating serum lipoprotein into the surrounding tissue. An isolated intracranial xanthoma is rarely reported and usually misdiagnosed. Intracranial xanthoma is also rarely found in patients with hyperlipidemia. To the best of the authors' knowledge, no previous studies and literature have reported bilateral involvement of intracranial xanthoma in the frontal lobe.

OBSERVATIONS The authors reported an unusual case of bilateral involvement of intracranial xanthoma in the frontal lobe with associated type II hyperlipidemia in a 42-year-old woman. Macroscopically, the tumor had an appearance of greyish-yellow color with a brittle, solid consistency. Histopathological examination revealed numerous lipid-laden macrophages surrounded by a cystic, necrotic, partially hemorrhagic area, with some parts consisting of hemosiderophages and proliferative capillary blood vessels. The histopathological findings indicated the characteristics of xanthoma.

LESSONS Bilateral frontal intracranial xanthoma with associated type II hyperlipidemia is an unusual finding. Despite its rarity and wide variety of radiological presentations, it should be considered one of the differential diagnoses of lesions that develop intracranially and intraaxially. Confirmation with histopathological examination is needed to exclude from other differential diagnoses.

https://thejns.org/doi/abs/10.3171/CASE21465

KEYWORDS intracranial tumor; intracranial xanthoma; neurosurgery; oncology

Xanthoma is a granulomatous lesion that develops as a result of leakage and accumulation of serum lipoprotein from the circulatory system into the surrounding tissue. ${ }^{1,2}$ Although it is commonly manifested superficially in the exposed surface as a subcutaneous xanthoma, xanthelasma, or arcus cornea, an isolated intracranial xanthoma is an unusual finding and is usually misdiagnosed. ${ }^{2-4}$ Because of its rarity, there is no exact prevalence of this lesion, which is only reported in some cases. Intracranial xanthoma is also rarely found in patients with hyperlipidemia, although the most common occurrence is in patients with type 1 I. $^{3,4}$ To the best of the authors' knowledge, no previous studies or literature has reported bilateral involvement of intracranial xanthoma in the frontal lobe.

We present a case report of bilateral frontal intracranial xanthoma that developed in a 42-year-old woman with associated type II hyperlipidemia. We provide a detailed description of the clinical course, physical and supportive examinations, histopathological findings, and management strategies as well as a review of this case based on previously reported studies and literature.

\section{Illustrative Case \\ History}

A 42-year-old woman presented to the neurosurgery clinic with a 2-year history of recurrent seizures. Her seizures are characterized by shaking all the extremities and being unconscious during each attack. They develop at least two to three times a week and last approximately 5 to 10 minutes. The patient also reports severe chronic headaches since 4 years ago, which feel like pressure of a heavy load in her head, especially over the forehead region. There are no symptoms such as nausea or vomiting, behavioral disturbance, weakness, sensory complaints, or

ABBREVIATIONS CT = computed tomography; FLAIR = fluid-attenuated inversion recovery; MRI = magnetic resonance imaging. INCLUDE WHEN CITING Published November 15, 2021; DOI: 10.3171/CASE21465. SUBMITTED August 14, 2021. ACCEPTED October 1, 2021.

* D.A. and C.L. share first authorship of this work.

(C) 2021 The authors, CC BY-NC-ND 4.0 (http://creativecommons.org/licenses/by-nc-nd/4.0/). 
presence of bumps in any parts of the body. The patient has a history of uncontrolled hyperlipidemia. No history of xanthoma was found in any of the patient's relatives.

\section{Examination}

The visual analog scale and Karnofsky performance scale ratings were 6 and 80 , respectively. No abnormalities were found on general and neurological examinations. No manifestations of xanthoma were found, such as subcutaneous and tendon xanthoma, arcus cornea, or xanthelasma of the eyelid. The lipid profile shows a significant levels of serum low-density lipoprotein (183 $\mathrm{mg} / \mathrm{dL})$ and serum cholesterol (265 mg/dL), with a normal serum triglyceride level (194 mg/dL). Based on the patient's history, we suspected an underlying intracranial space-occupying mass lesion, so we referred the patient to another province with magnetic resonance imaging (MRI) because there are no such facilities in East Nusa Tenggara.

MRI was performed, wherein the contrast-enhanced T1weighted, unenhanced T2-weighted, and fluid-attenuated inversion recovery (FLAIR) sequences showed a focal mass with heterogeneous signal intensity in the frontal lobe of both hemispheres, especially on the right side (Fig. 1A-D). The mass consisted primarily of a cystic component with some vascularized and necrotic area, surrounded by edema. There was no significant enhancement with intravenous contrast administration. Multiple small cystic components extended between the fornix and corpus callosum (Fig. 1C and D). Based on characteristics of $\mathrm{MRI}$, we suspected a malignant glioma, oligodendroglioma, lymphoma, or other intraaxial lesion as the primary differential diagnoses. We performed a surgical procedure to help relieve the symptoms and obtain a histopathological analysis of the underlying mass.
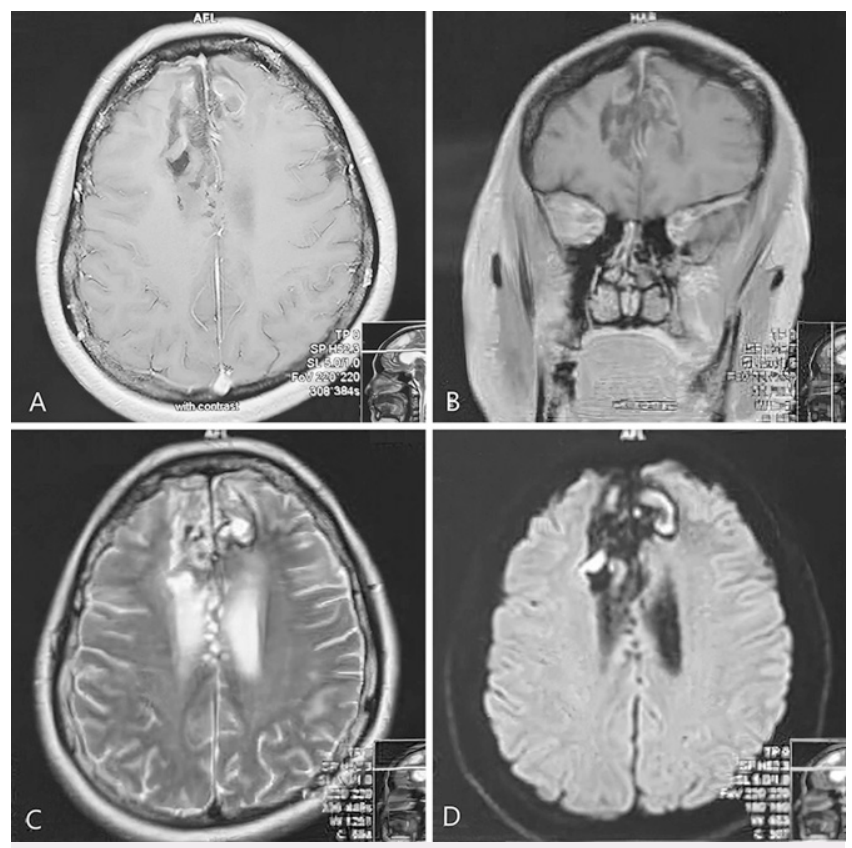

FIG. 1. MRI shows a focal mass with heterogeneous signal intensity in both frontal lobes, extending between the fornix and corpus callosum. A and B: Contrast-enhanced axial and coronal T1-weighted images. C: Unenhanced axial T2-weighted image. D: Axial FLAIR image.

\section{Operative Procedure}

We performed a horseshoe-shaped incision around the midline structures to expose both hemispheres. The lesions were approached interhemispherically, with high preservations of the superior sagittal sinus and draining cortical veins. We performed a subtotal resection of the mass in both frontal lobes, leaving unresectable structures between the fornix and corpus callosum. The mass was examined further at the pathology anatomy laboratory. The dura was then closed with a watertight closure technique and fibrin glue. The bone flap and skin were closed with sequential technique. The procedure lasted approximately 4 hours.

\section{Histopathologic Features}

We obtained two specimens, $1 \times 0.5 \times 0.3 \mathrm{~cm}$ and $5 \times 2 \times 2 \mathrm{~cm}$. Both specimens had a macroscopic greyish-yellow color with a brittle, solid consistency (Fig. 2). The microscopic appearance showed plenty of xanthoma cells characterized by numerous lipid-laden macrophages, surrounded by a cystic, necrotic, and hemorrhagic area (Fig. 2). Some parts consisted of hemosiderophages and proliferative capillary blood vessels. No evidence of malignancy was found in either specimen. Based on the histopathological findings, both specimens showed characteristics of intracranial xanthoma. The specimen was examined with hematoxylin and eosin stain (original magnification $\times 100$ ).

\section{Postoperative Management}

We obtained a postoperative contrast computed tomography (CT) scan, and the lesions in both frontal lobes were completely resected with some residual masses around the fornix and corpus callosum. The patient was given a statin class drug (atorvastatin 20 $\mathrm{mg}$ ) and recommended lifestyle modifications to help control hyperlipidemia. She was discharged on postoperative day 6; during the days of observation, there were no associated symptoms such as seizure, headache, or new neurological deficit. She lives on a remote island in East Nusa Tenggara, so we can only follow up with her by phone. We have followed the patient for at least 5 years, during which period there have been no reports of new or recurrent symptoms, and she can perform daily activities without any limitations. There are no facilities to provide imaging modalities such as CT or MRI in nearby health care facilities, so any change of the residual mass cannot be ascertained.
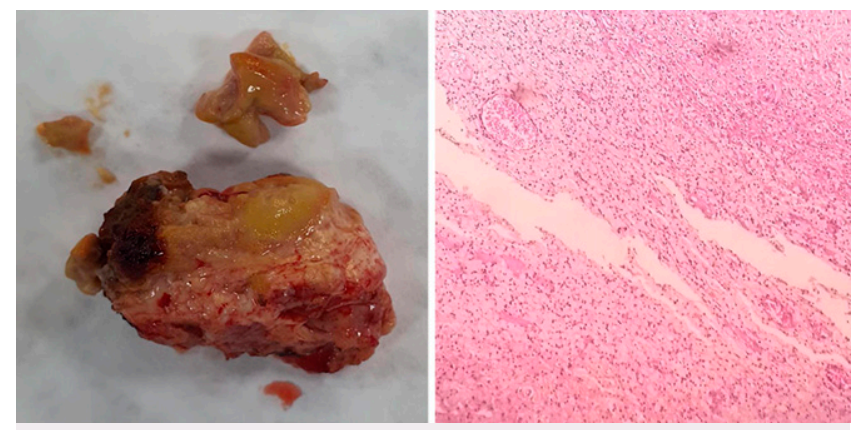

FIG. 2. The macroscopic appearance shows greyish-yellow masses with a brittle, solid consistency (left). The microscopic appearance shows numerous lipid-laden macrophages (hematoxylin and eosin stain; original magnification $\times 100$ ) (right). 


\section{Discussion \\ Observations}

Xanthoma is mainly associated with abnormal lipid storage or metabolism, which causes increased serum lipid level or hyperlipidemia. There are five types of hyperlipidemia; types II and III are caused by biochemical abnormalities that cause an increase in circulating lipoprotein with associated high serum cholesterol levels. In contrast, types I, IV, and V are caused by lipoprotein lipase deficiency and other glycoprotein abnormalities with associated high serum cholesterol and triglyceride levels. ${ }^{3,4}$ Intracranial xanthoma is rarely found in patients with hyperlipidemia, although the most common occurrence is in patients with type II. ${ }^{4,5}$ Development of intracranial xanthoma in the absence of hyperlipidemia can occur in histiocytic disorders such as xanthoma disseminatum. ${ }^{6}$

The development of xanthoma begins with leakage of serum lipoprotein and cholesterol from the circulatory system into the surrounding tissue, which activates the macrophages to phagocytize it. However, lipoprotein and cholesterol content cannot be degraded, thus forming a lipid-laden macrophage containing high lipid levels within the cytoplasm. The extracellular cholesterol levels that are not phagocytized by macrophages will crystalize and induce an inflammatory process that causes fibrosis. ${ }^{1,2}$ Xanthoma is frequently found in areas around the Achilles tendon, patella, hand extensor, and other exposed areas such as the cornea, eyelid, hand crease, elbow, and buttocks. ${ }^{5,7}$ The most common manifestations are subcutaneous and tendon xanthoma, arcus cornea, and xanthelasma of the eyelid., ${ }^{3,7}$ There is no known direct involvement of genetic or familial factors in developing intracranial xanthoma but they increase the chance of hyperlipidemia as one of the risk factors for xanthoma formation. ${ }^{4}$

Isolated intracranial xanthoma has rarely been reported and usually presents extraaxially in the calvaria or skull base or around the convexity. ${ }^{1,4,7-9}$ Intraaxially, some cases have involved xanthoma development in either the supra- or infratentorial compartment. ${ }^{2,10}$ Intracranial xanthoma rarely develops bilaterally. Based on reported studies and literature, there have been no cases of bilateral involvement of intracranial xanthoma in the frontal lobe. Signs and symptoms varied based on where the lesions grew, beginning with headache, seizures, behavioral changes, otorrhea, tinnitus, otalgia, or cranial nerve deficit. In our case, we reported an intracranial intraaxial xanthoma that developed bilaterally in the frontal lobe, and to the best of the authors' knowledge, this is the first reported case.

In general, the MRI characteristics of intracranial xanthoma resemble a high lipid content of the lesions. On unenhanced T1weighted images, most lesions show high signal intensity, followed by low signal intensity on T2-weighted images. ${ }^{2,4}$ There is a variable enhancement with intravenous contrast administration. ${ }^{2}$ In our case, contrast T1-weighted images show no significant enhancement, followed by heterogenous signal intensity on T2-weighted and FLAIR images in both frontal lobes, with some extension to the area between the fornix and corpus callosum. These findings do not show characteristics of xanthoma but rather show similar imaging features of other lesions around the frontal lobe, such as malignant glioma, oligodendroglioma, other primary brain tumors, or metastatic processes.

Histopathological examination is the main modality for diagnosing intracranial xanthoma, mainly because of its various imaging features and unpredictability due to the rarity of this lesion. In our reported case, we found a greyish-yellow mass with a brittle, solid consistency, which showed microscopic features of numerous lipidladen macrophages surrounded by a cystic, necrotic, partially hemorrhagic area and some parts that consisted of hemosiderophages and proliferative capillary blood vessels. Based on the literature and reported studies, these histopathological findings show characteristics of xanthoma. ${ }^{1,2,4,7-12}$ Some immunohistochemical markers, such as CD1a, S-100, glial fibrillary acidic protein, CD30, and CD21, could be used to differentiate from other underlying causes, such as Langerhans cell histiocytosis, glioma, follicular dendritic cell sarcoma, and lymphoma. ${ }^{2,13}$ However, no facilities exist to perform this examination at our institution.

Management of intracranial xanthoma consists of conservative medical management and surgical debulking. ${ }^{1,2,4,5,7,8,11}$ Conservative management to control underlying hyperlipidemia is recommended, although the correlation between high lipid levels and the development of xanthoma is not fully understood. Lifestyle modifications such as increasing physical activity and restricting high saturated fats and cholesterol in the diet, combined with pharmacological agents such as statins, niacin, and bile resin class drugs, provide significant control of hyperlipidemia. ${ }^{2-5,11}$ Surgical debulking in intracranial xanthoma was used to obtain a histopathological specimen for further examination and improve symptoms. ${ }^{1,4,7,8,10,11}$ However, the surgical procedure is often difficult to perform because of the complexity of anatomy surrounding the lesion growth, and it is often diagnosed late, so a lesion may expand and complicate a gross total resection. Any residual mass from a lesion presents a possibility of recurrence. ${ }^{4,10,14}$ In this case, our patient's condition was managed with a combination of medical and surgical approaches. We performed a subtotal resection and left a residual mass around the area between the fornix and corpus callosum. № follow-up imaging data are available, so any change of the residual mass cannot be ascertained.

\section{Lessons}

Bilateral frontal intracranial xanthoma with associated type II hyperlipidemia is an unusual finding. Despite its rarity and wide variety of radiological presentations, it should be considered one of the differential diagnoses of lesions that developed intracranially and intraaxially. Histopathological examination is the main modality for confirming diagnosis of intracranial xanthoma. Control of underlying hyperlipidemia and early recognition of this lesion are needed, especially to avoid extensive lesion growth to prevent gross total resection. We have some limitations, such as no immunohistochemistry examination and limited follow-up imaging data. Our patient lives on a remote island in East Nusa Tenggara with no such facilities to provide imaging, so any change of the residual mass cannot be ascertained.

\section{References}

1. Jackler RK, Brackmann DE. Xanthoma of the temporal bone and skull base. Am J Otol. 1987;8(2):111-115.

2. Hwang YJ, Hur G, Cha SJ, Kim YH, Kim SY, Kim MK. Intracranial xanthoma: long-term follow-up MR findings. AJNR Am J Neuroradiol. 2006;27(2):423-426.

3. Oberman A, Kreisberg R, Henkin Y, Sawin C. Principles and Management of Lipid Disorders: A Primary Care Approach. Lippincott-Raven; 1994.

4. Bonhomme GR, Loevner LA, Yen DM, Deems DA, Bigelow DC, Mirza N. Extensive intracranial xanthoma associated with type II hyperlipidemia. AJNR Am J Neuroradiol. 2000;21(2):353-355. 
5. Emery PJ, Gore M. An extensive solitary xanthoma of the temporal bone, associated with hyperlipoproteinaemia. J Laryngol Otol. 1982;96(5):451-457.

6. Chepuri NB, Challa VR. Xanthoma disseminatum: a rare intracranial mass. AJNR Am J Neuroradiol. 2003;24(1):105-108.

7. Basavaraj A, Jadhav S, Dhadwad J. Familial hypercholesterolemia presenting as intracranial xanthoma. J Assoc Physicians India. 2006;54:330-332.

8. Muthusamy KA, Azmi K, Narayanan P, Rajagopalan R, Rahman NA, Waran V. Bilateral temporal bone xanthoma. Case report. J Neurosurg. 2008;108(2):361-364.

9. Friedman O, Hockstein N, Willcox TO Jr, Keane WM. Xanthoma of the temporal bone: a unique case of this rare condition. Ear Nose Throat J. 2000;79(6):433-436.

10. Kasliwal MK, Suri A, Rishi A, Suri V, Sharma BS, Sarkar C. Symptomatic bilateral cerebellar mass lesions: an unusual presentation of intracranial xanthogranuloma. J Clin Neurosci. 2008;15(12):1401-1404.

11. Algoed L, Caemaert J, Achten E, van Aken J, de Reuck J. A large intracranial xanthoma in familial hypercholesterolemia. Clin Neurol Neurosurg. 1994;96(1):79-82.

12. Akazawa S, Ikeda Y, Toyama K, Miyake S, Takamori M, Nagataki S. Familial type lla hyperlipoproteinemia associated with a huge intracranial xanthoma. Arch Neurol. 1984;41(7):793-794.
13. Yang GZ, Li J, Wang LP. Disseminated intracranial xanthoma disseminatum: a rare case report and review of literature. Diagn Pathol. 2016;11(1):78.

14. Ferlito A, Recher G, Bordin S. Involvement of the temporal bone in hyperlipidemic xanthomatosis. Otolaryngol Head Neck Surg. 1983;91(1):100-104.

\section{Disclosures}

The authors report no conflict of interest concerning the materials or methods used in this study or the findings specified in this paper.

\section{Author Contributions}

Conception and design: all authors. Acquisition of data: Lauren, Argie. Analysis and interpretation of data: Lauren, Argie. Drafting the article: all authors. Critically revising the article: all authors. Reviewed submitted version of manuscript: Lauren, Argie. Approved the final version of the manuscript on behalf of all authors: Lauren. Statistical analysis: Lauren, Argie. Administrative/technical/material support: all authors. Study supervision: all authors. Operating surgeon: Argie.

\section{Correspondence}

Christopher Lauren: Prof. Dr. W. Z. Johannes Kupang Regional General Hospital, Kupang, East Nusa Tenggara, Indonesia. chrislauren11@gmail.com. 